\title{
Spectral Line Broadening in Dense Plasmas
}

\author{
Banaz Omar \\ Institut für Physik, Universität Rostock, Universitätsplatz 3, 18051 Rostock, Germany \\ Correspondence should be addressed to Banaz Omar, b.omar@dr.com
}

Received 20 October 2010; Accepted 25 January 2011

Academic Editor: Elliot P. Kanter

Copyright (C) 2011 Banaz Omar. This is an open access article distributed under the Creative Commons Attribution License, which permits unrestricted use, distribution, and reproduction in any medium, provided the original work is properly cited.

\begin{abstract}
Spectral line broadening is calculated based on a microscopic quantum statistical approach. By using thermodynamic Green's function, plasma correlation effect, electrostatic and dynamic screening, and perturber-radiator interaction are taken into account. Ions are treated in quasistatic approximation due to Stark effect. The line broadening for $6678 \AA\left(2^{1} \mathrm{P}-3^{1} \mathrm{D}\right)$ and $5016 \AA\left(2^{1} \mathrm{~S}\right.$ $\left.3^{1} \mathrm{P}\right)$ transitions of neutral helium is calculated in the electron density range $n_{e}=(0.25-50) \times 10^{22} \mathrm{~m}^{-3}$ and temperature range $T=(0.5-4) \times 10^{4} \mathrm{~K}$, and the density and temperature dependence of the line width are investigated. A good agreement is shown by comparing the calculated values with the existing experimental and theoretical data.
\end{abstract}

\section{Introduction}

Optical spectroscopy is one of the most important diagnostic tools to characterize warm and dense plasmas. The emitted radiation from a plasma is perturbed by interaction between a radiating atom and surrounding particles, which leads to spectral line broadening; the most effective mechanism is Stark broadening (pressure broadening). Line profile calculation is an interesting technique to determine the internal plasma parameters, such as density, temperature, and composition, to study the microscopic processes within plasma, and to check the quality of the predicted parameters $[1,2]$.

Several semiclassical and quantum-mechanical approaches have been investigated to calculate spectral line shapes in plasmas [1, 3-16]. Helium lines were calculated by Griem et al. $[1,3]$ in a semiclassical approach, the so-called standard theory (ST), using an impact approximation for electrons with a cut-off procedure, while ions were treated in a quasistatic approximation due to the static microfield of perturbers. The electron broadening impact parameters are calculated for neutral helium lines by using semiclassical perturbation formalisms based on the approach developed by Sahal-Bréchot $[4,5]$. Furthermore, the convergent theory was improved by Bassalo et al. [6] in a many-level approximation to calculate the Stark broadening parameters of neutral helium lines. Recently, molecular dynamics (MD) simulations have been performed by Calisti et al. [17] and Gigosos et al. $[18,19]$ by including the time sequence of the microfield distribution as a random process. The quantummechanical Green's function method is considered in this paper to calculate the He I spectral lines in dense plasmas, assuming local thermal equilibrium (LTE) [20-26].

Spectral line profiles of helium are important in plasma diagnostic. Some of the lines are measured in a pulsed arc plasma by Pérez et al. [27-29], studied in the astrophysical context, for example, stellar atmospheres of hot stars and white dwarfs [30-33], and in the He-atmosphere evaluation of hot stars of types O and B [34]. Helium spectral lines have been studied previously; the $6678 \AA$ line is of importance to determine physical properties of massive compact binaries [35]. Furthermore, the He I $6678 \AA$ line is measured from optical spectroscopic observation of CI Cam [36] and the dwarf nova GW Librae [37]. The He I line emission at $5016 \AA$ was detected in the central region of a starburst galaxy Tol 1924-416 [38] and in Mrk 110 [39]. Spectra in the visible range (4100-7100) $\AA$ have been obtained for a sample of main sequence B-type stars to determine the behavior of neutral helium lines with effective temperature and gravity [34].

In Section 2 an overview of the spectral line modeling is given. Result and discussion are shown in Section 3. The comparison with other theoretical and experimental results is presented. Finally, conclusion is given in Section 4 . 


\section{Theoretical Approach of Line Broadening in Dense Plasmas}

A systematic account of medium modifications of the absorption coefficient $\alpha(\omega)$ is possible using the dielectric approach which links the absorption and emission coefficients to the dielectric tensor. For visible light, the absorption coefficient is given by the long-wavelength limit of the dielectric function $\varepsilon(\omega)$

$$
\alpha(\omega)=\frac{\omega}{c n(\omega)} \operatorname{Im} \varepsilon(\omega),
$$

where $n(\omega)$ is the index of refraction which in turn reads

$$
n(\omega)=2^{-1 / 2}\left\{\operatorname{Re} \varepsilon(\omega)+\left[(\operatorname{Re} \varepsilon(\omega))^{2}+(\operatorname{Im} \varepsilon(\omega))^{2}\right]^{1 / 2}\right\}^{1 / 2} .
$$

The microscopic treatment of the line shapes in dense plasmas starts form the dielectric approach given by (1) and (2). Neglecting change in the index of refraction, the cluster expansion of the dielectric function together with a systematic perturbative analysis using thermodynamic Green's function shows that the spectral line shape is proportional to the Fourier transform of the dipoledipole autocorrelation function [24]. The perturber-radiator interaction leads to a pressure broadening, which contains electronic and ionic contributions. Describing the ionic contribution in the quasi-static approximation by averaging over the ionic microfield $[24,25]$, we have

$$
\begin{aligned}
& I^{\mathrm{pr}}(\omega) \\
& \sim \sum_{i^{\prime}, i^{\prime \prime}, f^{\prime}, f^{\prime \prime}}\left\langle i^{\prime}|\mathbf{r}| f^{\prime}\right\rangle\left\langle f^{\prime \prime}|\mathbf{r}| i^{\prime \prime}\right\rangle \frac{\omega^{4}}{8 \pi^{3} c^{3}} e^{-\hbar \omega / k_{\mathrm{B}} T} \int_{0}^{\infty} W(\beta) d \beta \\
& \times \operatorname{Im}\left\langle i^{\prime}\left|\left\langle f^{\prime}\left|\left[\hbar \omega-\hbar \omega_{i f}-\Sigma_{i f}(\omega, \beta)+i \Gamma_{i f}^{\mathrm{v}}\right]^{-1}\right| f^{\prime \prime}\right\rangle\right| i^{i^{\prime}}\right\rangle,
\end{aligned}
$$

where $\langle i|\mathbf{r}| f\rangle$ is identified as a dipole matrix element for the transition between initial $i$ and final $f$ states. The ionic microfield distribution function $W(\beta)$ is taken according to the Hooper microfield distribution function with field strength $\beta=E / E_{0}$ normalized to the Holtsmark field $E_{0}$ [49]. Equation $\hbar \omega_{i f}=E_{i}-E_{f}$ is the unperturbed transition energy.

The function $\Sigma_{i f}(\omega, \beta)$ is determined by the self-energy correction $\Sigma_{n}(\omega, \beta)$ of the initial $(n=i)$ and the final $(n=f)$ states

$$
\begin{aligned}
\Sigma_{i f}(\omega, \beta)= & \operatorname{Re}\left[\Sigma_{i}(\omega, \beta)-\Sigma_{f}(\omega, \beta)\right] \\
& +i \operatorname{Im}\left[\Sigma_{i}(\omega, \beta)+\Sigma_{f}(\omega, \beta)\right] .
\end{aligned}
$$

Both electronic and ionic contributions occur in the selfenergy

$$
\Sigma_{n}(\omega, \beta)=\Sigma_{n}^{\mathrm{ion}}(\beta)+\Sigma_{n}^{\mathrm{el}}(\omega, \beta) .
$$

Performing Born approximation with respect to the dynamically screened perturber-radiator potential, the electronic self-energy is obtained as [24]

$$
\begin{aligned}
\Delta_{n}^{\mathrm{SE}}+ & i \Gamma_{n}^{\mathrm{SE}} \\
= & \left\langle n\left|\Sigma^{\mathrm{el}}\left(E_{n}, \beta\right)\right| n\right\rangle=-\frac{1}{e^{2}} \int \frac{\mathrm{d}^{3} q}{(2 \pi)^{3}} V(q) \sum_{\alpha}\left|M_{n \alpha}(\mathbf{q})\right|^{2} \\
& \times \int_{-\infty}^{\infty} \frac{\mathrm{d} \omega}{\pi}\left[1+n_{\mathrm{B}}(\omega)\right] \frac{\operatorname{Im} \varepsilon^{-1}(\mathbf{q}, \omega+i 0)}{E_{n}-E_{\alpha}(\beta)-\hbar(\omega+i 0)} .
\end{aligned}
$$

Here, the level splitting $\left(E_{\alpha}(\beta) \approx E_{\alpha}\right)$ due to the microfield has been neglected [23], $n_{\mathrm{B}}(\omega)=\left[\exp \left(\hbar \omega / k_{\mathrm{B}} T\right)-1\right]^{-1}$ is the Bose distribution function, and $M_{n \alpha}(\mathbf{q})$ is the transition matrix-element given in the following. The sum over $\alpha$ runs from $n-2$ to $n+2$ discrete bound states for the virtual transitions. Dynamical screening effect is accounted for in (6) from imaginary part of the inverse dielectric function $\varepsilon^{-1}(\mathbf{q}, \omega)$

$$
\operatorname{Im} \varepsilon^{-1}(\mathbf{q}, \omega)=-\frac{\operatorname{Im} \varepsilon(\mathbf{q}, \omega)}{[\operatorname{Re} \varepsilon(\mathbf{q}, \omega)]^{2}+[\operatorname{Im} \varepsilon(\mathbf{q}, \omega)]^{2}} .
$$

The dielectric function is approximated by random phase approximation (RPA) as

$$
{ }_{\varepsilon^{\mathrm{RPA}}}(\mathbf{q}, \omega)=1-2 V(q) \int \frac{\mathrm{d}^{3} p}{(2 \pi)^{3}} \frac{f_{e}\left(E_{\mathbf{p}}\right)-f_{e}\left(E_{\mathbf{p}+\mathbf{q}}\right)}{E_{\mathbf{p}}-E_{\mathbf{p}+\mathbf{q}}-\hbar(\omega+i 0)},
$$

where $E_{p}=\hbar^{2} \mathbf{p}^{2} /\left(2 m_{e}\right)$ is the kinetic energy of electron. The Fermi distribution function $f_{e}\left(E_{\mathbf{p}}\right)$ of electrons in the nondegenerate limit can be approximated by Boltzmann distribution function

$$
f_{e}\left(E_{\mathbf{p}}\right) \simeq \frac{1}{2} n_{e}\left(\frac{2 \pi \hbar^{2}}{m_{e} k_{\mathrm{B}} T}\right)^{3 / 2} \exp \left(-\frac{\hbar^{2} p^{2}}{2 m_{e} k_{\mathrm{B}} T}\right) .
$$

Binary collision approximation can be considered, which leads to a linear behavior of the electronic width and shift with respect to the electron density

$$
\operatorname{Im} \varepsilon^{-1}(\mathbf{q}, \omega) \approx-\operatorname{Im} \varepsilon(\mathbf{q}, \omega) .
$$

Considering Born approximation, the electronic part is overestimated; to avoid this we apply the cut-off procedure and add the strong collision term [1] in state of partial summation of the three-particle T-matrix, where the result might be slightly modified $[24,26]$.

The transition matrix element $M_{n \alpha}(\mathbf{q})$ describes the interaction of the atom with the Coulomb potential through the vertex function. In lowest order, it can be determined by the atomic eigenfunctions $\psi_{n}(\mathbf{P})$. The Coulomb interaction with electron-electron-ion triplet depends on the momentum transfer $\hbar \mathbf{q}$. For helium and hydrogen atoms, the matrix 
elements can be represented by the following diagrams [20, 22]:

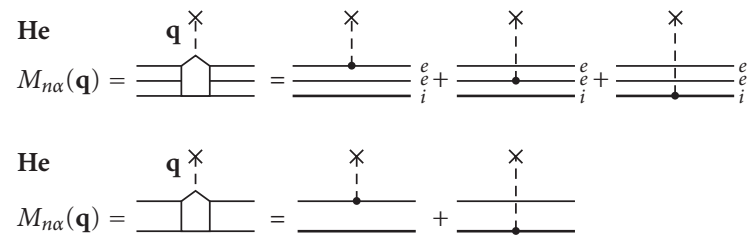

However, the matrix element of helium can be approximated by the one of hydrogen, while the outer electron is screened by inner electron. The matrix-element of hydrogen reads

$$
\begin{aligned}
M_{n \alpha}(\mathbf{q}) & =\int \frac{\mathrm{d}^{3} p}{(2 \pi)^{3}} \psi_{n}^{*}(\mathbf{p}) \\
& \times\left[Z e \psi_{\alpha}\left(\mathbf{p}-\frac{m_{e}}{m_{i}+m_{e}} \mathbf{q}\right)-e \psi_{\alpha}\left(\mathbf{p}+\frac{m_{i}}{m_{i}+m_{e}} \mathbf{q}\right)\right],
\end{aligned}
$$

assuming that the ion with effective charge $Z$ is much heavier than the electron $m_{i} \gg m_{e}$

$$
\begin{aligned}
M_{n \alpha}(\mathbf{q}) & \approx \int \frac{\mathrm{d}^{3} p}{(2 \pi)^{3}} \psi_{n}^{*}(\mathbf{p})\left[Z e \psi_{\alpha}(\mathbf{p})-e \psi_{\alpha}(\mathbf{p}+\mathbf{q})\right], \\
& \approx i e\left(Z \delta_{n \alpha}-\int \mathrm{d}^{3} r \psi_{n}^{*}(\mathbf{r}) \exp (i \mathbf{q} \cdot \mathbf{r}) \psi_{\alpha}(\mathbf{r})\right) .
\end{aligned}
$$

Expanding the plane wave into spherical harmonics

$$
\exp (i \mathbf{q} \cdot \mathbf{r})=4 \pi \sum_{l=0}^{\infty} \sum_{m=-l}^{l} i^{l} j_{l}(q r) Y_{l m}^{*}\left(\Omega_{q}\right) Y_{l m}\left(\Omega_{r}\right),
$$

where $j_{l}(q r)$ is the spherical Bessel function, a multipole expansion can be derived, and, for example, $l=0,1,2$ gives the monopole, dipole, and quadrupole contributions of the radiator-electron interaction; respectively.

The vertex correction $\Gamma_{i f}^{\mathrm{v}}$ for the overlapping line is related to coupling between the initial and the final states, given by $[20,24]$

$$
\begin{aligned}
\Gamma_{i f}^{\mathrm{v}}= & -\frac{4 \pi}{e^{2}} \int \frac{\mathrm{d}^{3} q}{(2 \pi)^{3}} \frac{\mathrm{d}^{3} p}{(2 \pi)^{3}} f_{e}\left(E_{\mathbf{p}}\right) V^{2}(q) \\
& \times M_{i i}(\mathbf{q}) M_{f f}(-\mathbf{q}) \delta\left(\frac{\hbar^{2} \mathbf{p} \cdot \mathbf{q}}{m_{e}}\right) .
\end{aligned}
$$

In static limit, static Debye screening can be considered appropriate for the inverse dielectric function such as $[23$, 50]

$$
\begin{gathered}
\operatorname{Im} \varepsilon^{-1}(\mathbf{q}, \omega)=-\frac{\operatorname{Im} \varepsilon(\mathbf{q}, \omega)}{\left(1+\left(\kappa_{\mathrm{D}}^{2} / q^{2}\right)\right)^{2}}, \\
\varepsilon^{\mathrm{RPA}}(\mathbf{q}, \omega \longrightarrow 0)=1+\frac{\kappa_{\mathrm{D}}^{2}}{q^{2}},
\end{gathered}
$$

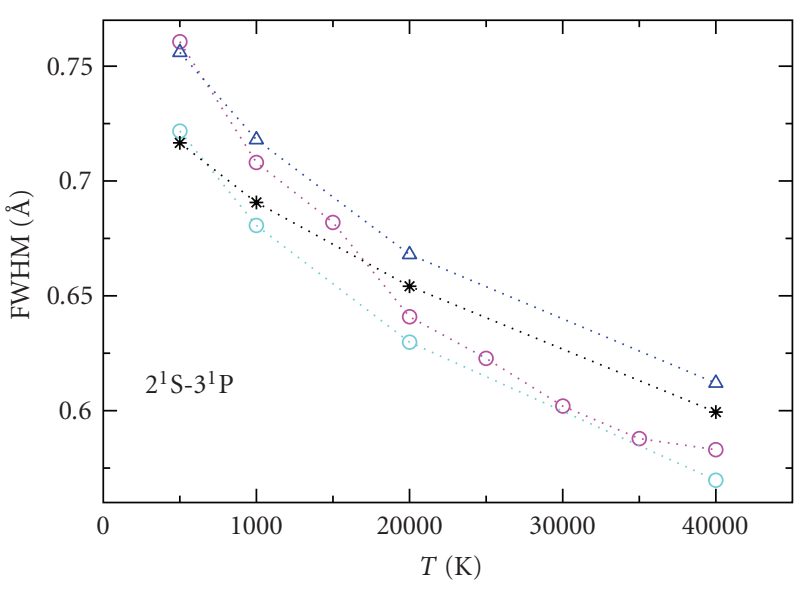

๑. Gigosos et al., independent particles
.$\quad$ Gigosos et al., interacting particles
$\triangle$. Griem (1974)
.$*$ This work

Figure 1: The electronic FWHM $\left(2 w_{e}\right)$ of He I $5016 \AA$ line versus temperature at electron density $n_{e}=1.0 \times 10^{22} \mathrm{~m}^{-3}$. Comparison is made with the impact approximation [1] and the MD simulations data $[18,19]$.

where $\kappa_{\mathrm{D}}=r_{\mathrm{D}}^{-1}=\left[2 e^{2} n_{e} /\left(\epsilon_{0} k_{\mathrm{B}} T\right)\right]^{1 / 2}$ is the inverse Debye radius. However, this approximation is valid for virtual transition between states with negligible energy difference.

For nonhydrogenic radiator, ionic contribution to the self-energy is related to quadratic Stark effect and quadrupole interaction, further detail is given in $[25,26]$ The microfield can be considered as a static microfield distribution function, while it does not change during the time of interest for the radiation process. The full line profile is obtained by convolution of pressure broadening with Gaussian distribution of Doppler broadening [51]

$$
\begin{gathered}
I(\omega)=\int_{\infty}^{\infty} I^{\mathrm{D}}(\omega-\bar{\omega}) I^{\mathrm{pr}}(\bar{\omega}) \mathrm{d} \bar{\omega} \\
I(\omega) \sim \int_{-\infty}^{\infty} \frac{d \bar{\omega}}{\bar{\omega}} \exp \left[-\frac{m_{i} c^{2}}{2 k_{\mathrm{B}} T}\left(\frac{\omega-\bar{\omega}}{\bar{\omega}}\right)^{2}\right] I^{\mathrm{pr}}(\bar{\omega}),
\end{gathered}
$$

where $m_{i}$ is the mass of the radiating atom.

\section{Result and Discussion}

In this section, we present the profiles and the full widths at half maximum (FWHM) of the $5016 \AA$ and $6678 \AA$ lines by applying the approach outlined above, assuming LTE. The comparison of available data and our calculated results is shown in this section.

The full electronic width $\left(2 w_{e}\right)$ for the lines $5016 \AA$ and $6678 \AA$ are calculated from thermodynamic Green's function, shown in Figures 1 and 2, respectively. The electronic width is compared with the results of the ST of Griem [1] and of the MD simulations of Gigosos et al. [18, 19]. Furthermore, in Figure 2 the semiclassical calculation of Dimitrijević and Sahal-Bréchot [30] and the result of Bassalo et al. [6] 
are also included. The MD simulations [18, 19] results correspond to two different kinds of MD simulations, in the first calculation the independent electrons moving with a constant speed along straight line trajectories. The correlation between particles is considered by using a screened Coulomb field. The second case is for interacting particles in a one-component plasma, and a regularized potential is used for close collision. The electron-ion interaction is attractive; therefore, configurations involving electrons at distance of the order of de Broglie wavelength or shorter have to be considered. The Coulomb interaction must be modified in a classical description at such distances. The ion-electron potential regularization provides well-defined classical physics for opposite sign charge systems, and allows the application of the various sophisticated classical many-body methods of classical statistical mechanics [52]. Moreover, the correlation arises, so the interaction with the emitter is simply the Coulomb potential $[18,19]$. Figures 1 and 2 show the interaction as well as the correlation between perturbers, they are more important for decreasing temperature. The discrepancy between both simulations results can be seen at low temperature. Our results in Figure 2 give rather smaller values than the others, especially at low temperature. This might be due to degeneracy in the plasma. The contribution of strong collision to the width is rather small for these two lines, about $20 \%$ at $5000 \mathrm{~K}$. Furthermore, the dynamically screened Coulomb interaction is included.

The measured profile of the line $5016 \AA$ was recorded by Soltwisch and Kusch [40] in a wall-stabilized pulsed discharge plasma at electron density $n_{e}=7.7 \times 10^{22} \mathrm{~m}^{-3}$ and temperature $T=20000 \mathrm{~K}$. The comparison is made with the ST of Griem and his collaborators $[1,3]$ taken from the [40]; see Figure 3. Our calculations are also included, and the best agreement is achieved in the case of statical screening effect. The discrepancies at the far wings can be related to the perturbing neighbouring lines.

In Figure 4, the FWHM of He I for the transition line $5016 \AA$ is plotted versus the electron density. The comparison is made with large number of experimental results and theoretical calculations. This line was investigated by Berg et al. [42]; electromagnetic shock fronts were employed as a thermal light source. Electron densities were determined from continuum intensity and temperature from intensity ratios of ion and neutral lines. One of the most interesting measurements of Stark broadening of visible neutral helium lines in plasmas has been carried out by Kelleher [46], where sixteen different spectral lines emitted from a wallstabilized arc have been studied in detail. Stark broadening of several isolated neutral helium lines such as $5016 \AA$ and $6678 \AA$ emitted from dense plasmas has been measured in a low-pressure, pulsed arc plasma by Pérez et al. [27]. The electron density was determined by interferometry for different wavelengths, and the plasma electron temperature was estimated from a Boltzmann plot or the intensity ratio of the ion and neutral lines. The results of the experimental Stark broadening of these two lines in a lowpressure, pulsed arc $\mathrm{H}-\mathrm{He}$ plasma have been presented by Mijatović et al. [47]. The electron density is measured by laser interferometry. The electron temperature is determined

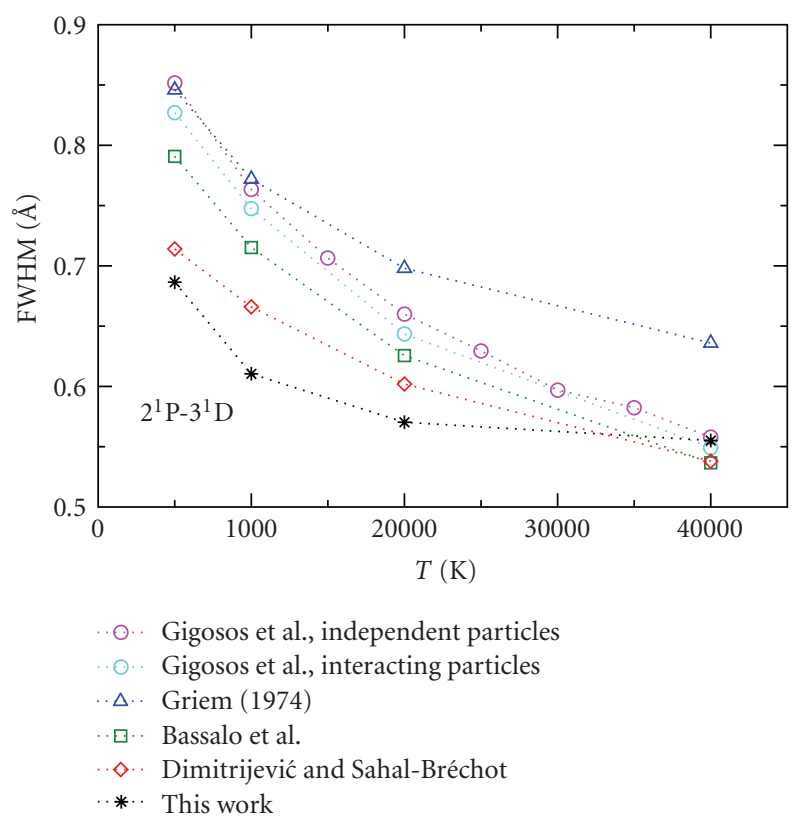

FIgure 2: Total electronic width $\left(2 w_{e}\right)$ of the He I $6678 \AA$ line as function of temperature at electron density $n_{e}=1.0 \times 10^{22} \mathrm{~m}^{-3}$. Comparison is made with the other theoretical data $[1,6,18,19$, 30].

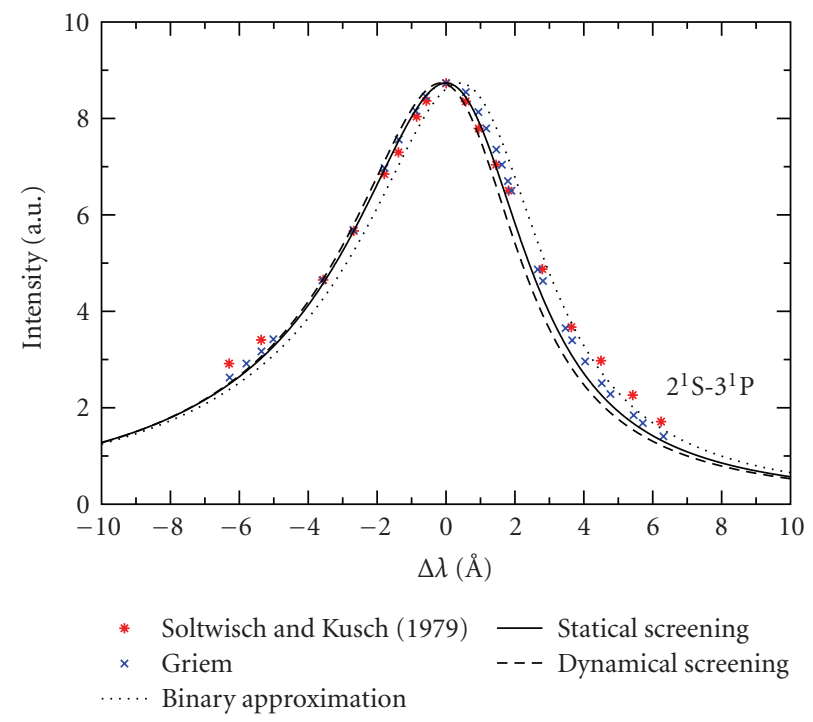

FIgURE 3: Experimental and theoretical line profiles of He I $5016 \AA$ at electron density $n_{e}=7.7 \times 10^{22} \mathrm{~m}^{-3}$ and temperature $T=$ $20000 \mathrm{~K}[1,40]$.

from the intensity ratio of the $\mathrm{H}$-line to the underlying continuum, while the gas temperature is measured from the Doppler broadening [45]. Furthermore, the transition lines have been measured by Milosavljević and Djeniže $[31,45]$ at electron density between $(0.3-8.2) \times 10^{16} \mathrm{~cm}^{-3}$ and electron temperature between $(8-33) \times 10^{3} \mathrm{~K}$. Plasmas were created under five various discharge conditions using a linear low-pressure pulsed arc as an optically thin plasma 


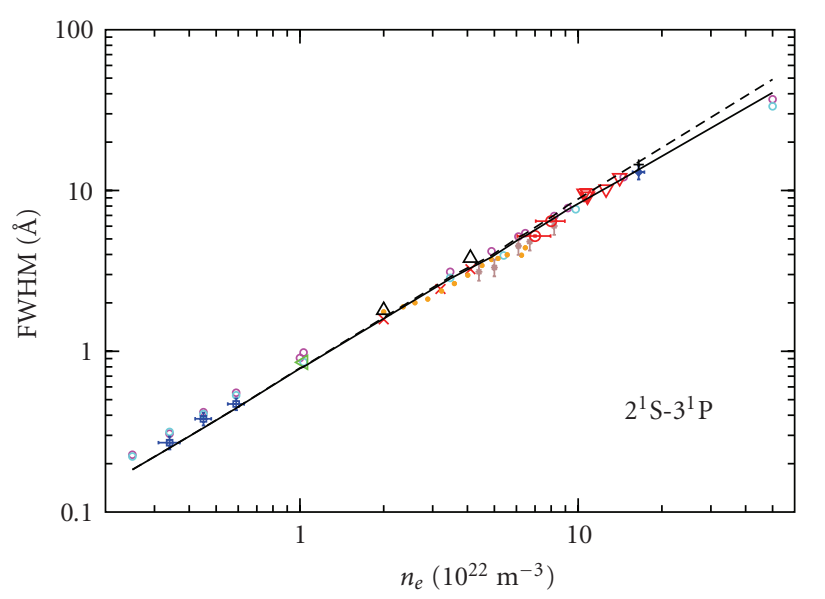

- Gigosos et al., independent particles

Gigosos et al., interacting particles

$\times$ Bassalo et al.

- Berg et al.

Pérez et al.

$\nabla \quad$ Chiang et al.

$\Delta$ Diatta

Milosavljević and Djeniže

$\triangleleft$ Kelleher

Mijatović et al.

- Soltwisch and Kusch

+ Griem

- - - Binary approximation

- Dynamical screening

Figure 4: Stark FWHM for the He I $5016 \AA$ line as a function of the electron density. A large number of measured and calculated data are included. In this paper the line broadening for the $\left(2^{1} \mathrm{~S}-3^{1} \mathrm{P}\right)$ transition is calculated by using binary approximation $(---)$ and including dynamical screening effect ( - ). They are compared with the data of Gigosos et al. for pink circle (०) independent and turquoise circle $(0)$ interacting particles $[18,19],(\times)$ Bassalo et al. [41], $(\diamond)$ Berg et al. [42], orange circle $(\bullet)$ Pérez et al. [27], $(\nabla)$ Chiang et al. [43], $(\triangle)$ Diatta [44], (*) Milosavljević and Djeniže [45], $(\triangleleft)$ Kelleher [46], ( $\square)$ Mijatović et al. [47], ( $\bigcirc)$ Soltwisch and Kusch [40], and (+) Griem [1].

source operated in a helium-nitrogen-oxygen gas mixture. Also the measurements of Diatta [44] and Chiang et al. [43] are included. Our approach shows a good agreement with the other results. Still some discrepancy can be observed at very low densities, where the dynamical motion of ion is considered in the MD simulation. At very high electron densities the binary collision approximation overestimates the width.

The spectral line shape of the line $6678 \AA$ is measured by Vujičić et al. [48] in the laser-produced plasma at electron density $n_{e}=10.6 \times 10^{22} \mathrm{~m}^{-3}$ and temperature $T=3.1 \times$ $10^{4} \mathrm{~K}$, where the validity of the quasi-static approximation is reasonable with the exception of the line center. The reduced line profile [48] was compared with the ST [1], obtained by averaging electron impact parameters over the static ion microfield strength. We also include our calculated profile $J(x)$ as function of reduced wavelength $x$; see $[1,48]$. The

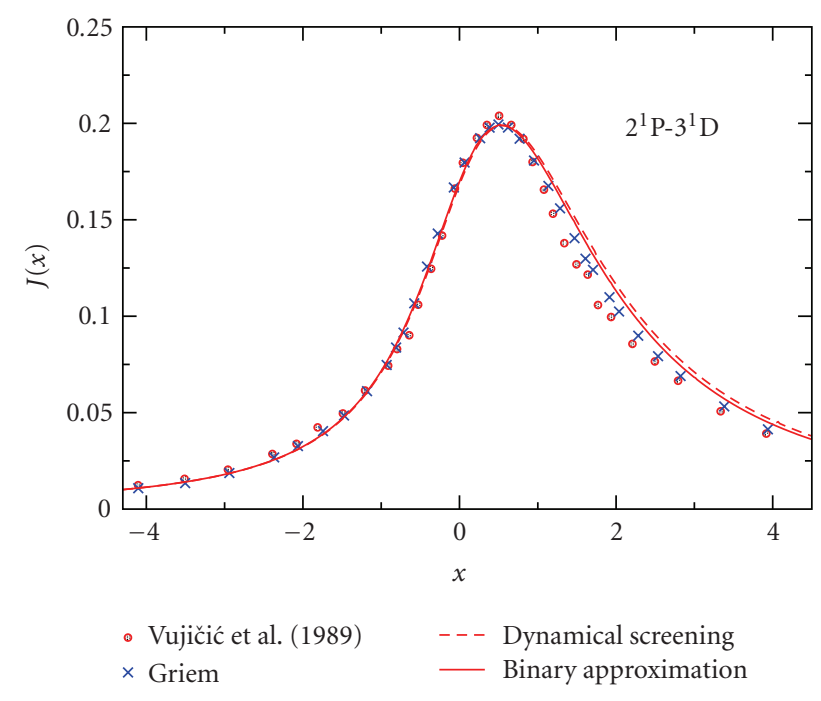

FIGURE 5: Comparison of the measured and theoretical line shapes for the He I $6678 \AA$ line in reduced wavelength at temperature $T=$ $31000 \mathrm{~K}$ and electron density $n_{e}=10.6 \times 10^{22} \mathrm{~m}^{-3}[48]$.

comparison is already shown in Figure 5, and asymmetry of the line profiles can be observed. The overall agreement can be seen between our calculations and the other profiles.

Furthermore, in Figure 6 the FWHM calculation is performed for the line $6678 \AA$. Additional results for a larger range of density are shown: values obtained by Pérez et al. [27] for electron density $(2.0-6.46) \times 10^{22} \mathrm{~m}^{-3}$ and temperature of $(1.9-4.3) \times 10^{4} \mathrm{~K}$, experimental values by Mijatović et al. [47], Kelleher [46], and measurement by Milosavljević and Djeniže [31]. A comparison with the theoretical result by Bassalo et al. [41] is given as well. Furthermore, the results of MD simulations $[18,19]$ in the density range of $(0.25-50.0) \times 10^{22} \mathrm{~m}^{-3}$ and temperature range of $(1.9-4.2) \times 10^{4} \mathrm{~K}$ are included. The theoretical scaling data of ST is taken from Pérez et al. [27]. The estimated width of ST [1] is systematically higher than our result. The Doppler broadening becomes less important with increasing density, and the width is only due to Stark broadening at high density, this can be seen in Figure 6 . In general, our calculated FWHM result for the given density and temperature agrees with the other theoretical and experimental values.

The inclusion of dynamical screening reduces the magnitude of width at high electron density, causing some nonlinear behavior, see Figures 4 and 6 . Regarding the data reported by Bassalo et al. [41], the discrepancy may be due to neglecting the width in lower energy states of transition. They use the Debye radius for the maximum impact parameter and take the reduced matrix element from the oscillator strength of transition. Our approach shows a good agreement with the other results. Still some discrepancy can be observed at very low electron density where the dynamical motion of ions is more pronounced. The quadratic Stark effect increases slightly the line width and shift, and introduces asymmetric ion broadening component to the line shape. However, the ion contribution is relatively 


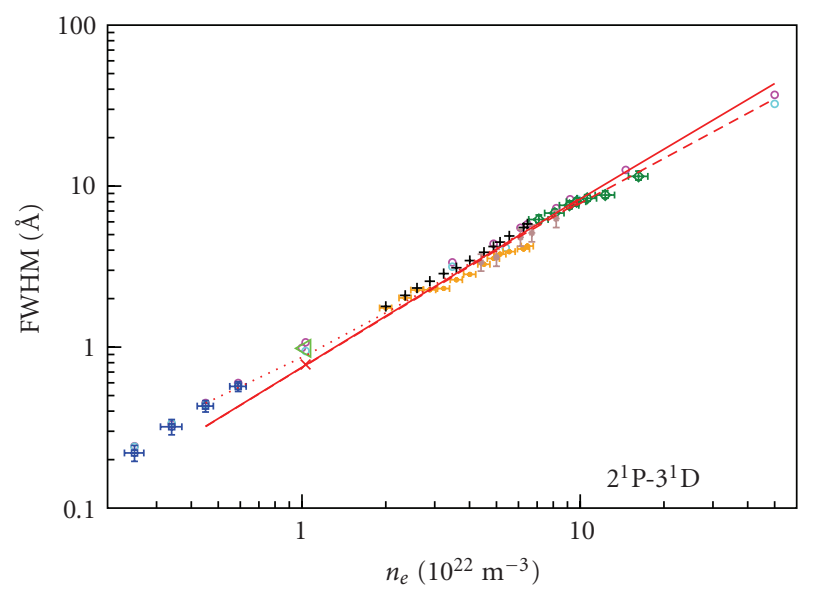

- Gigosos et al., independent particles

Gigosos et al., interacting particles

$\times$ Bassalo et al.

$\triangleleft$ Kelleher

Pérez et al.

- Mijatović et al.

$\diamond$ Vujičić et al.

- Milosavljević and Djeniže

+ Griem

This work, with Doppler

— This work, binary approximation

- - - This work, dynamical screening

FIgURE 6: The full width at half maximum of the He I line $6678 \AA$ versus electron density. Our FWHM calculations for this line by including Doppler broadening $(. . . \cdot)$, binary approximation $(-)$, and dynamical screening effect $\left(--_{-}\right)$are compared with results of Gigosos et al. for pink circle (०) independent and turquoise circle $(\circ)$ interacting particles $[18,19],(\times)$ Bassalo et al. [41], $(\triangleleft)$ Kelleher [46], orange circle $(\bullet)$ Pérez et al. [27], ( $\square$ )

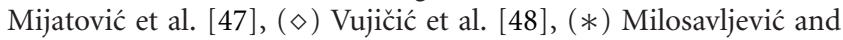
Djeniže [31], and (+) Griem [1].

high for the He I $6678 \AA$ and $5016 \AA$ lines they are both extremely asymmetric see Figures 3 and 5 . With increasing electron density, the binary collision approximation is no longer valid, and plasma oscillations become relevant, where the dynamical screening effect modifies the broadening parameters with respect to the density [53-55]. Then, the exact expression of the inverse dielectric function has to be used, especially when the interacting transition frequency becomes comparable or smaller than the electron plasma frequency. The lines might be no longer isolated, or the linear Stark effect might be of importance at high electron density.

\section{Conclusion}

In this study, the quantum statistical approach is presented to the line profile calculation of allowed isolated He I $6678 \AA$ and $5016 \AA$ lines; however, this method is applicable for both allowed and forbidden radiative transitions. A cut off procedure is used for strong collisions. The Coulomb approximation is employed to evaluate the wavefunctions of helium atom. The dynamical screening effect increases at high density, leading to nonlinear behavior of Stark parameters with increasing density $n_{e}$. In our calculation, the time-dependent microfield fluctuation is approximated by its static value due to the large mass of the ion relative to the electron mass, assuming the stationary motion of ions during the time of collisions. The contribution of ions is taken into account in the quasi-static approximation, with both quadratic Stark effect and quadrupole interaction. Good agreement can be clearly seen by comparing our result with the existing data.

\section{Acknowledgment}

The author would like to thank S. Günter, A. Wierling, and G. Röpke for helpful discussions.

\section{References}

[1] H. R. Griem, Spectral Line Broadening by Plasmas, Academic Press, New York, NY, USA, 1974.

[2] W. Lochte-Holtgreven, Plasma Diagnostics, North-Holland, Amsterdam, The Netherlands, 1968.

[3] H. R. Griem, M. Baranger, A. C. Kolb, and G. Oertel, "Stark broadening of neutral helium lines in a plasma," Physical Review, vol. 125, no. 1, pp. 177-195, 1962.

[4] S. Sahal-Bréchot, "Impact theory of the broadening and shift of spectral lines due to electrons and ions in a plasma," Astronomy \& Astrophysics, vol. 1, pp. 91-123, 1969.

[5] S. Sahal-Bréchot, "Impact theory of the broadening and shift of spectral lines due to electrons and ions in a plasma (continued)," Astronomy \& Astrophysics, vol. 2, pp. 322-354, 1969.

[6] J. M. Bassalo, M. Cattani, and V. S. Walder, "Convergent calculations for electron impact broadening and shift of neutral helium lines," Journal of Quantitative Spectroscopy and Radiative Transfer, vol. 28, no. 2, pp. 75-80, 1982.

[7] A. C. Kolb and H. Griem, "Theory of line broadening in multiplet spectra," Physical Review, vol. 111, no. 2, pp. 514-521, 1958.

[8] M. Baranger, "Spectral line broadening in plasmas," in Atomic and Molecular Processes, D. R. Bates, Ed., chapter 13, Academic Press, New York, NY, USA, 1962.

[9] H. Margenau and M. Lewis, "Structure of spectral lines from plasmas," Reviews of Modern Physics, vol. 31, no. 3, pp. 569-615, 1959.

[10] E. W. Smith, J. Cooper, and C. R. Vidal, "Unified classical-path treatment of Stark broadening in plasmas," Physical Review, vol. 185, no. 1, pp. 140-151, 1969.

[11] R. W. Lee, "Plasma line shapes for selected transitions in hydrogen-, helium- and lithium-like ions," Journal of Quantitative Spectroscopy and Radiative Transfer, vol. 40, no. 5, pp. 561-568, 1988.

[12] M. Baranger, "General impact theory of pressure broadening," Physical Review, vol. 112, no. 3, pp. 855-865, 1958.

[13] D. Voslamber, "Unified quantum statistical formulation of pressure broadening," Physics Letters A, vol. 40, no. 3, pp. 266-268, 1972.

[14] T. Hussey, J. W. Dufty, and C. F. Hooper Jr., "Kinetic theory of spectral line broadening," Physical Review A, vol. 12, no. 3, pp. 1084-1093, 1975.

[15] D. B. Boercker and J. W. Dufty, "Quantum kinetic theory of time-correlation functions," Physical Review A, vol. 23, no. 4, pp. 1952-1968, 1981. 
[16] H. Nguyen, M. Koenig, D. Benredjem, M. Caby, and G. Coulaud, "Atomic structure and polarization line shift in dense and hot plasmas," Physical Review A, vol. 33, no. 2, pp. 1279-1290, 1986.

[17] A. Calisti, R. Stamm, and B. Talin, "Simulation calculation of the ion-dynamic effect on overlapping neutral helium lines," Physical Review A, vol. 38, no. 9, pp. 4883-4886, 1988.

[18] M. A. Gigosos, M. A. Gonzalez, B. Talin, and A. Calisiti, "Molecular dynamics simulations of He I Stark broadened line profiles," in Proceedings of the 17th International Conference on Spectral Line Shapes, E. Dalimier, Ed., p. 451, Frontier Group, Paris, France, 2004.

[19] M. A. Gigosos, M. A. Gonzalez, B. Talin, and A. Calisiti, unpublished.

[20] W.-D. Kraeft, D. Kremp, W. Ebeling, and G. Röpke, Quantum Statistics of Charged Particle Systems, Akademie, Berlin, Germany, 1986.

[21] S. Günter, "Stark shift and broadening of hydrogen spectral lines," Contributions to Plasma Physics, vol. 29, no. 4-5, pp. 479-487, 1989.

[22] S. Günter, L. Hitzschke, and G. Röpke, "Hydrogen spectral lines with the inclusion of dense-plasma effects," Physical Review A, vol. 44, no. 10, pp. 6834-6844, 1991.

[23] S. Günter and A. Könies, "Quantum mechanical electronic width and shift of spectral lines over the full line profileelectronic asymmetry," Journal of Quantitative Spectroscopy and Radiative Transfer, vol. 52, no. 6, pp. 819-824, 1994.

[24] S. Günter, Optische Eigenschaften dichter Plasmen [Habilitation thesis], Rostock University, Rostock, Germany, 1966.

[25] S. Sorge, A. Wierling, G. Röpke, W. Theobald, R. Sauerbrey, and T. Wilhein, "Diagnostics of a laser-induced dense plasma by hydrogen-like carbon spectra," Journal of Physics B, vol. 33, no. 16, pp. 2983-3000, 2000.

[26] B. Omar, S. Günter, A. Wierling, and G. Röpke, "Neutral helium spectral lines in dense plasmas," Physical Review E, vol. 73, no. 5, Article ID 056405, 2006.

[27] C. Pérez, I. de la Rosa, A. M. de Frutos, and S. Mar, "Calibration of the Stark-broadening parameters for some He i lines," Physical Review A, vol. 44, no. 10, pp. 6785-6790, 1991.

[28] C. Pérez, R. Santamarta, M. I. de la Rosa, and S. Mar, "Stark broadening of neutral helium lines and spectroscopic diagnostics of pulsed helium plasma," European Physical Journal D, vol. 27, no. 1, pp. 73-75, 2003.

[29] C. Pérez, J. A. Aparicio, I. de la Rosa, S. Mar, and M. A. Gigosos, "Calibration of the Stark-broadening parameters of the 728.1-nm He i line," Physical Review E, vol. 51, no. 4, pp. 3764-3766, 1995.

[30] M. S. Dimitrijević and S. Sahal-Bréchot, "Stark broadening of neutral helium lines," Journal of Quantitative Spectroscopy and Radiative Transfer, vol. 31, no. 4, pp. 301-313, 1984.

[31] V. Milosavljević and S. Djeniže, "Ion contribution to the astrophysical important 447.15, 587.56 and $667.82 \mathrm{~nm} \mathrm{He}$ I spectral lines broadening," Astronomy \& Astrophysics, vol. 393, no. 2, pp. 721-726, 2002.

[32] A. Beauchamp, F. Wesemael, and P. Bergeron, "Spectroscopic studies of DB white dwarfs: improved Stark profiles for optical transitions of neutral helium," Astrophysical Journal, Supplement Series, vol. 108, no. 2, pp. 559-573, 1997.

[33] M. S. Dimitrijević, "On the variation of Stark line widths within a supermultiplet," Astronomy \& Astrophysics, vol. 112, no. 2, pp. 251-256, 1982.
[34] F. Leone and A. C. Lanzafame, "Visible neutral helium lines in main sequence B-type stars: observations and NLTE calculations," Astronomy \& Astrophysics, vol. 330, no. 1, pp. 306-310, 1998.

[35] J. A. Harvin, D. R. Gies, W. G. Bagnuolo Jr., L. R. Penny, and M. L. Thaller, "Tomographic separation of composite spectra. VIII. The physical properties of the massive compact binary in the triple star system HD 36486 ( $\delta$ Orionis A)," Astrophysical Journal, vol. 565, no. 2, pp. 1216-1230, 2002.

[36] J. Yan, Q. Liu, and H. Hang, "Optical spectroscopic observations of CI Camelopardalis," Astronomical Journal, vol. 133, no. 4, pp. 1478-1484, 2007.

[37] K. Hiroi, Y. Moritani, D. Nogami et al., "Spectroscopic observations of the WZ Sge-type dwarf nova GW librae during its 2007 superoutburst," Publications of the Astronomical Society of Japan, vol. 61, no. 4, pp. 697-705, 2009.

[38] M. Iye, M.-H. Ulrich, and M. Peimbert, "High resolution spectrum of the starburst galaxy tololo 1924-416," Astronomy \& Astrophysics, vol. 186, no. 1-2, pp. 84-94, 1987.

[39] W. Kollatschny, K. Bischoff, E. L. Robinson, W. F. Welsh, and G. J. Hill, "Short-term emission line and continuum variations in Mrk 110," Astronomy \& Astrophysics, vol. 379, no. 1, pp. 125-135, 2001.

[40] H. Soltwisch and H. J. Kusch, "Experimental Stark profile determination of some plasma broadened He II-lines," Zeitschrift Naturforschung Teil A, vol. 34, p. 300, 1979.

[41] J. M. Bassalo, M. Cattani, and V. S. Walder, "Semiclassical convergent calculations for the electron-impact broadening and shift of some lines of neutral helium in a hot plasma," Physical Review A, vol. 22, no. 3, pp. 1194-1197, 1980.

[42] H. F. Berg, A. W. Ali, R. Lincke, and H. R. Griem, "Measurement of Stark profiles of neutral and ionized helium and hydrogen lines from shock-heated plasmas in electromagnetic T tubes," Physical Review, vol. 125, no. 1, pp. 199-206, 1962.

[43] W. T. Chiang, D. P. Murphy, Y. G. Chen, and H. R. Griem, "Electron densities from Stark widths of the HeI $5016 \AA$ and HeI $3889 \AA$ lines and helium-neon laser interferometry," Zeitschrift Naturforschung Teil A, vol. 32, p. 818, 1977.

[44] C. S. Diatta, Ph.D. thesis unpublished, University d'Orleans, Paris, France, 1977.

[45] V. Milosavljević and S. Djeniže, "Ion contribution to the astrophysical important 388.86, 471.32 and $501.56 \mathrm{~nm} \mathrm{He}$ I spectral lines broadening," New Astronomy, vol. 7, no. 8, pp. 543-551, 2002.

[46] D. E. Kelleher, "Stark broadening of visible neutral helium lines in a plasma," Journal of Quantitative Spectroscopy and Radiative Transfer, vol. 25, no. 3, pp. 191-220, 1981.

[47] Z. Mijatović, N. Konjević, M. Ivković, and R. Kobilarov, "Influence of ion dynamics on the width and shift of isolated He i lines in plasmas. II," Physical Review E, vol. 51, no. 5, pp. 4891-4896, 1995.

[48] B. T. Vujičić, S. Djurović, and J. Halenka, "The Stark broadening of the He I $667.8 \mathrm{~nm}$ line," Zeitschrift für Physik $D$, vol. 11, no. 2, pp. 119-121, 1988.

[49] C. F. Hooper Jr., "Low-frequency component electric microfield distributions in plasmas," Physical Review, vol. 165, no. 1, pp. 215-222, 1968.

[50] St. Böddeker, S. Günter, A. Könies, L. Hitzschke, and H. J. Kunze, "Shift and width of the $\mathrm{H} \alpha$ line of hydrogen in dense plasmas," Physical Review E, vol. 47, no. 4, pp. 2785-2791, 1993.

[51] D. Salzmann, Atomic Physics in Hot Plasmas, Oxford University Press, Oxford, UK, 1998. 
[52] E. Dufour, A. Calisti, B. Talin et al., "Charge-charge coupling effects on dipole emitter relaxation within a classical electronion plasma description," Physical Review E, vol. 71, no. 6, Article ID 066409, 9 pages, 2005.

[53] D. Kremp, M. Schlanges, and W.-D. Kraeft, Quantum Statistics of Nonideal Plasmas, Springer, Berlin, Germany, 2005.

[54] G. Röpke and L. Hitzschke, "Shift and broadening of spectral lines in non-ideal plasmas," in Proceedings of the 9th International Conference on Spectral Line Shapes, J. Szudy, Ed., p. 49, Ossolineum, Waroclaw, Poland, 1989.

[55] L. Hitzschke and S. Günter, "The influence of many-particle effects beyond the Debye approximation on spectral line shapes in dense plasmas," Journal of Quantitative Spectroscopy and Radiative Transfer, vol. 56, no. 3, pp. 423-441, 1996. 

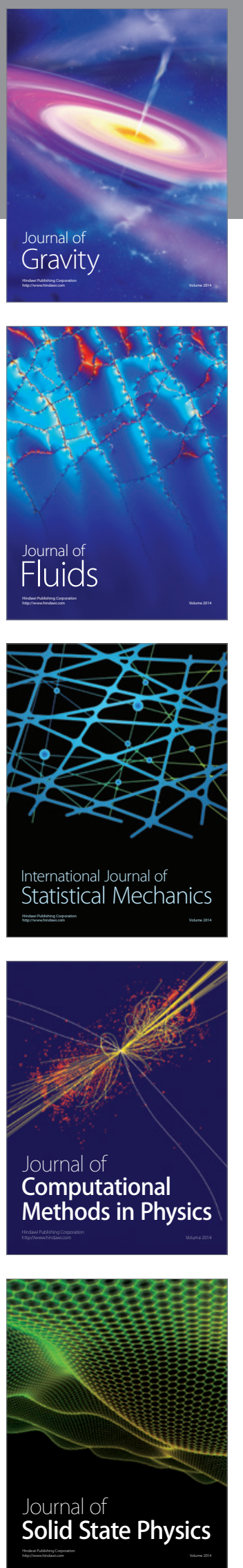

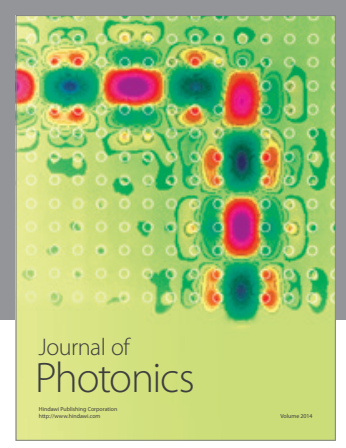

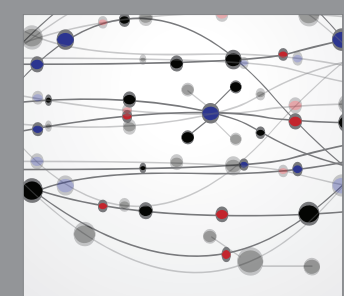

The Scientific World Journal
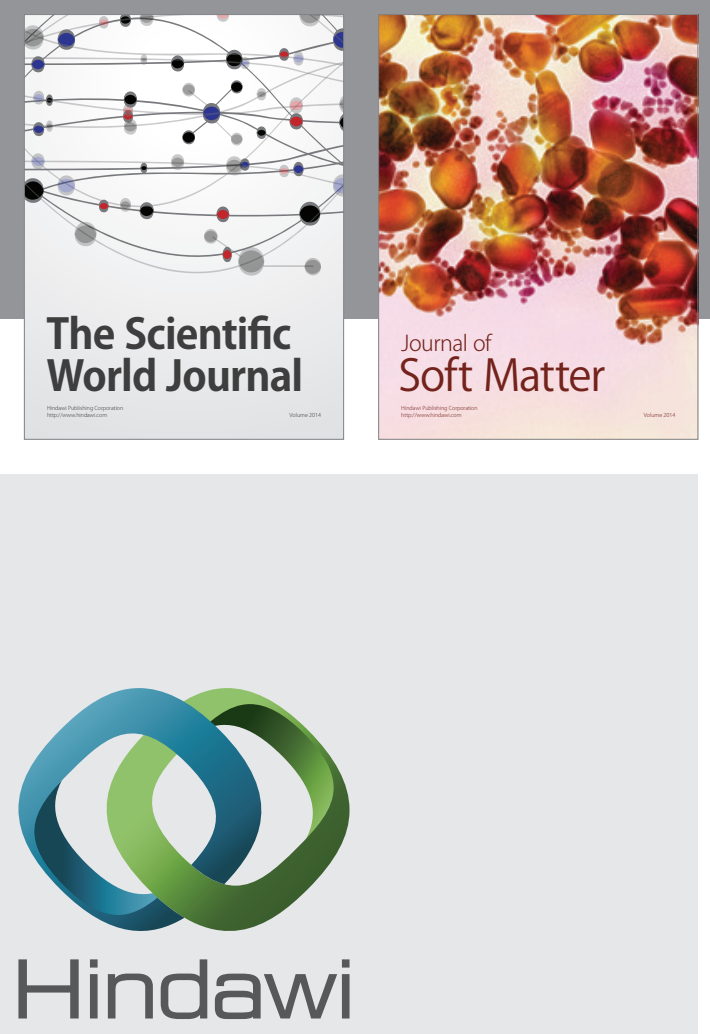

Submit your manuscripts at

http://www.hindawi.com
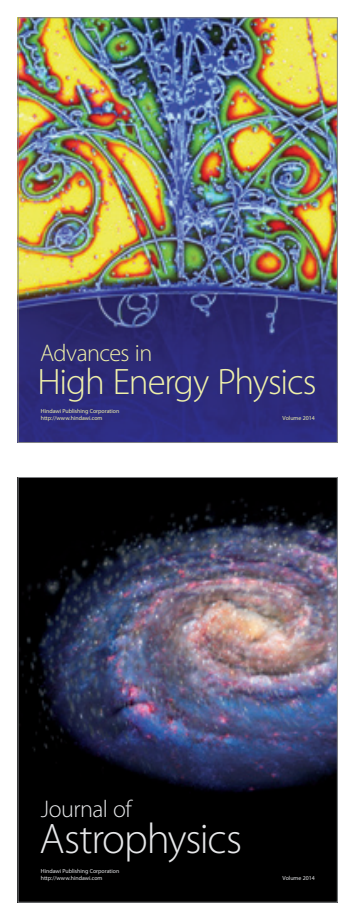
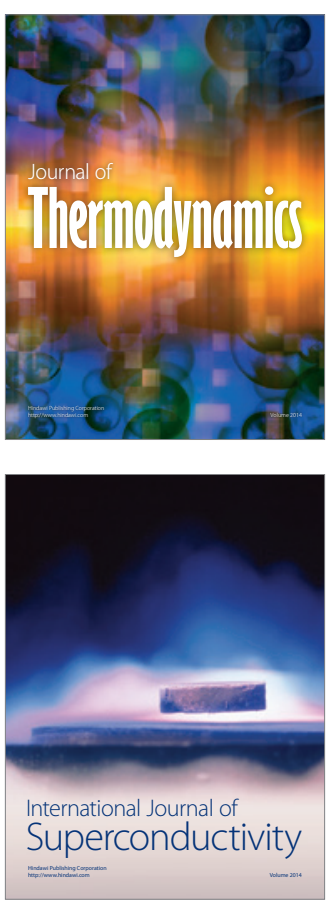
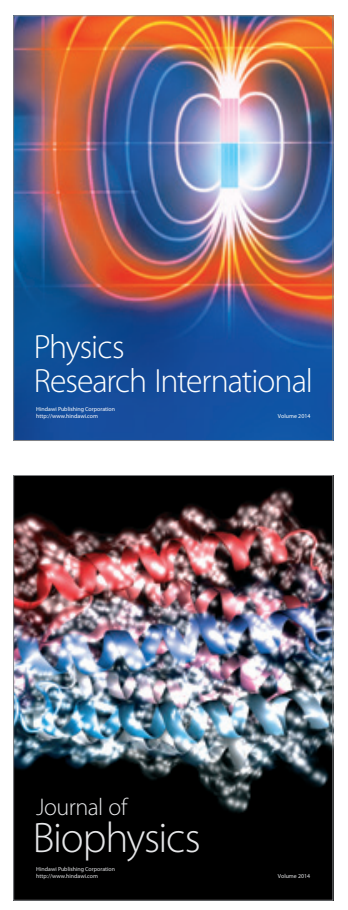
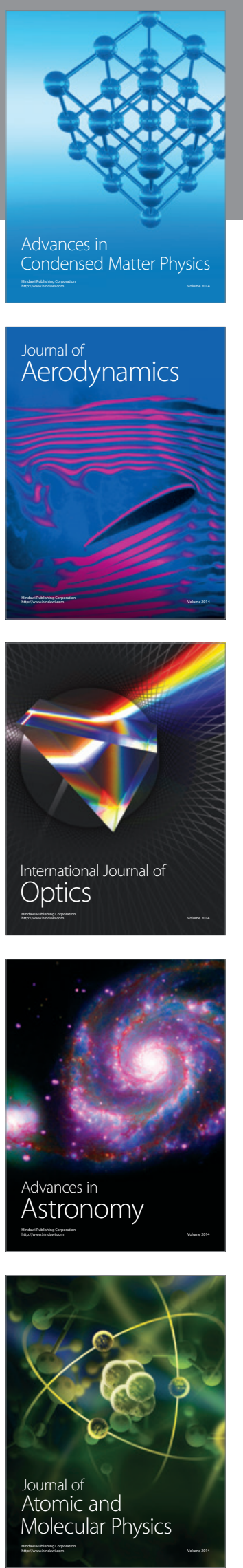